\title{
Pin-ning down p53 function
}

Cis-trans isomerization of peptide bonds involving proline is emerging as a relatively widespread mechanism for the reversible allosteric regulation of protein function. Site-specific prolyl isomerization is catalysed by a family of enzymes that are collectively known as peptidyl-prolyl isomerases (PPI).

The PPI Pin-1 regulates a growing number of proteins involved in cellular proliferation in a phosphorylationdependent manner. Substrate recognition is mediated by the interaction of an amino-terminal 'WW' domain, a conserved phosphoprotein-binding module, with a phosphorylated phospho-Ser/Thr-Pro motif in the target protein, resulting in cis-trans isomerization of this peptide bond.

Two recent papers in Nature (Zacchi, P. et al. Nature 419, 853-857 (2002) and Zheng, H. et al. Nature 419, 849-853 (2002)) have now enriched the repertoire of targets for Pin-1 by demonstrating that DNA-damage-induced activation of the tumour suppressor p53 is mediated by Pin- 1 . Both studies show that Pin-1 interacts directly with p53 in a manner that is dependent on genotoxic-stress-induced phosphorylation at three previously characterized sites on the tumour suppressor. Zacchi et al. show that Pin1-induced isomerization of p53 results in decreased binding to the E3 ubiquitin ligase $\mathrm{Mdm} 2$ and hence stabilization of the p53 protein, at least in some cellular settings. p53 functions primarily as a transcription factor, and Pin-1-induced isomerization results in increased binding of p53 to consensus enhancer sites and increased transactivation of target-genes (including the Cdk inhibitor $p 21^{\text {Wafl }}, M d m 2$ and the pro-apoptotic genes Bax and Killer/DR5), partially through stabilization of p53, but possibly also through direct effects on these activities. Evidently, a detailed molecular mechanistic understanding awaits further study.

Pin-1-knockout mice have restricted proliferative defects and both groups demonstrate that Pin-1-null cells have defects in the

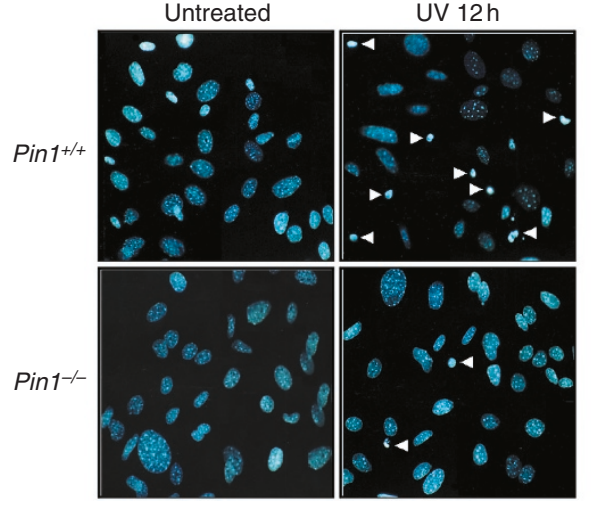

Figure 1 p53-mediated apoptosis is dependent on Pin1. Pin1 $1^{+/+}$ and Pin $1^{-/}$MEFs were treated with UV. Representative fields of the nuclear morphology after UV irradiation are shown.

accumulation and activation of p53 after DNA damage. Consequently, they suffer from defects in both cell cycle checkpoint controls and apoptosis in response to DNA damage.

p53 governs the response to DNA damage by cell cycle arrest or apoptosis and its activity is highly controlled at multiple levels, primarily through the regulation of protein stability. This data adds a new facet to the increasingly complex array of mechanisms that regulate the stability of p53 in response to stress.

BERND PULVERER

\section{Drosophila, actin and videotape - new insights in wound healing}

Stéphane Noselli

The combined use of laser ablation and live imaging in Drosophila melanogaster embryos provides new ways to visualize the dynamics of wound healing. Analysis of lesions made in living embryos mutant for small GTPases reveal distinct and unexpected roles for actin cables and protrusions in wound repair. Hole closure is definitely more complex than simply pulling a string.

W ound healing has been a recurrent challenge throughout the course of human history and probably involved a diverse array of strategies aimed at producing the perfect heal. Nonetheless, the clinical and aesthetic consequences of wounds are often the cause of concern and frustration. To help design the ideal band- age, the empirical approaches of the past are now being replaced by analytical methods in several cell and tissue models ${ }^{1}$, allowing us to unravel the complicated events underlying wound healing. However, a study by Wood et al. in this issue of Nature Cell Biology $y^{2}$ reports the reduction of a complex biological process down to a manageable model system, the Drosophila embryo. This may prove central to our understanding of the cell dynamics and cell signalling activities that accompany hole closure.

The attractiveness of Drosophila as a wound healing model stems from studies on dorsal closure ${ }^{3}$, a developmental process similar to wound healing (Fig. 1). Dorsal 